\title{
Staphylococcus aureus e Salmonella sp. em queijos de coalho artesanais produzidos em São Rafael, Rio Grande do Norte
}

\section{Staphylococcus aureus and Salmonella sp. in curd cheese produced in São Rafael, Rio Grande do Norte}

\author{
Tarciara Magley da Fonseca Pereira ${ }^{1 *}$, Vilson Alves de Góis ${ }^{2}$, Karoline Mikaelle de Paiva Soares ${ }^{3}$, Lara Barbosa de Souza ${ }^{4}$ \\ José Aldenor de Sousa ${ }^{5}$
}

\begin{abstract}
Resumo: O queijo de coalho artesanal é produto de origem animal muito consumido principalmente na região Nordeste do Brasil. É comumente fabricado com leite cru, não pasteurizado, como matéria prima, sem a utilização de boas práticas higiênicas, podendo veicular micro-organismos patogênicos ao consumidor. Assim, objetivou-se avaliar a presença de Salmonella sp. e quantificar Staphyloccocus aureus em queijos de coalho produzidos artesanalmente no município de São Rafael Rio Grande do Norte.Foram realizadas coletas em triplicata em sete queijarias artesanais do município, e, em seguida, os queijos foram transportados imediatamente em caixas isotérmicas, ao Laborátório de Biotecnologia Industrial da Universidade Federal Rural do Semi-Árido, onde foram submetidos à análise microbiológica de forma asséptica, conforme metodologia recomendada pela Instrução Normativa $n^{\circ}$ 62, de 26 de agosto de 2003, do Ministério da Agricultura, Pecuária e Abastecimento. Todas as amostras apresentaram elevadas contagens de Staphylococcus aureus, estando em desconformidade com o padrão exigido pela legislação brasileira para este grupo microbiano. Foi constatado ausência de Salmonella sp. nas amostras avaliadas. As análises microbiológicas evidenciaram péssimas condições de higiene de todos os queijos estudados, já que os mesmos apresentaram grande contaminação por Staphylococcus aureus, o que pode representar riscos à saúde pública.
\end{abstract}

Palavras-chave: Micro-organismos patogênicos; Higiene; Saúde pública.

\begin{abstract}
Artisanal coalho cheese is a product of animal origin consumed mainly in the northeastern region of Brazil. It is commonly made with raw, unpasteurized milk as raw material, without the use of good hygienic practices, and can transmit pathogenic microorganisms to the consumer. Thus, the present study aimed to evaluate the presence of Salmonella sp. And to quantify Staphylococcus aureus in rennet cheeses produced artisanally in the municipality of São Rafael-RN. Triplets were collected in triplicate in seven artisanal dairies of the municipality, and then the cheeses were transported immediately in isothermal boxes, to the Laboratory of Biotechnology Industrial of the Federal Rural University of the Semi-Arid, where they were submitted to the microbiological analysis aseptically, according to methodology recommended by Normative Instruction No. 62, of August 26, 2003 (MAPA). All samples presented high Staphylococcus aureus counts, being in disagreement with the standard required by Brazilian legislation for this microbial group. It was found absence of Salmonella sp. in the evaluated samples. Microbiological analyzes showed poor hygienic conditions for all the cheeses studied, since they presented a high level of contamination with Staphylococcus aureus, which may represent a risk to public health.
\end{abstract}

\footnotetext{
*Autor para correspondência

Recebido para publicação em 23/03/2017; aprovado em 15/06/2017

${ }^{1}$ Mestre em Ambiente, Tecnologia e Sociedade, Universidade Federal Rural do Semi-Árido, Mossoró, tarciara@ufersa.edu.br.

${ }^{2}$ Doutor em Fitotecnia, Laboratório de Tecnologia Agroindustrial, Centro de Ciências Agrárias, Universidade Federal Rural do Semi-Árido, vilsongois@ufersa.edu.br

${ }^{3}$ Doutora em Ciência Animal, Laboratório de Biotecnologia de Alimentos, Centro de Ciências Agrárias, Universidade Federal Rural do Semi-Árido, karolinesoares@ufersa.edu.br

${ }^{4}$ Doutoranda em Ciência Animal, Laboratório de Inspeção de Produtos de Origem Animal, Universidade Federal Rural do Semi-Árido, larabiotec@ gmail.com

${ }^{5}$ Mestre em Sistemas Agroindustriais, Laboratório de Tecnologia Agroindustrial, Centro de Ciências Agrárias, Universidade Federal Rural do Semi-Árido, josealdenor@ufersa.edu.br
} 


\section{INTRODUÇÃO}

O leite bovino é um alimento de alto valor nutricional, considerado isento de micro-organismos quando no interior do canal galactófaro de vacas sadias. Ao ser obtido, este alimento pode ser contaminado por uma variedade de microorganismos, sendo que sua composição facilita o crescimento microbiano (MACHADO; LIMA, 2010). O leite serve de matéria prima para uma grande variedade de produtos lácteos, sendo que o queijo é considerado o laticínio mais tradicional (SILVA; FERNANDES, 2011).

Para produção deste derivado lácteo, uma prática comum, é sua elaboração informal em propriedades rurais (SCHIMITT et al., 2011). Com destaque para o queijo coalho na região Nordeste (OLIVEIRA et al., 2010), onde muitas vezes é produzido com leite cru e sem a utilização de condições higiênico sanitárias satisfatórias (SOUSA et al., 2014). Salmonella sp. e Staphylococcus aureus são exemplos de micro-organismos patógenos de grande incidência em queijos que não são produzidos com condições adequadas de higiene (SANTANA et al., 2008).

A Salmonella sp. é a causa mais importante de doenças veiculadas por alimentos (GERMANO; GERMANO, 2008), enquanto o Staphylococcus está presente de forma frequente nas vias aéreas superiores do homem, podendo facilmente se disseminar para as mãos dos manipuladores, que por sua vez, contamina o alimento (JAY, 2005; FORSYTHE, 2013)

A Resolução da Diretoria Colegiada $n^{\circ} 12$ da Agência Nacional de Vigilância Sanitária estabelece os requisitos de qualidade microbiológica para alimentos. Autores vêm detectando a presença de patógenos em queijos de coalho (BORGES et al., 2003; FEITOSA et al., 2003; SOUSA et al., 2014). Para a produção de queijos de coalho seguros ao consumidor, é necessário o emprego de boas práticas durante a obtenção da matéria prima e processamento (MIRANDA et al., 2016). Assim, a tradição do queijo de coalho na região Nordeste e a preocupação com as condições higiênicosanitárias da produção e comercialização deste alimento por pequenos produtores demostra a importância da realização de pesquisas para determinação microbiológica desses produtos, com isso objetivou-se avaliar a ocorrência de Staphylococcus aureus e Salmonella sp. em queijos de coalho artesanais produzidos em queijarias no município de São Rafael no Estado do Rio Grande do Norte.

\section{MATERIAL E MÉTODOS}

A pesquisa foi realizada de Abril de 2014 a Maio de 2015, no município de São Rafael, Rio Grande do Norte (RN). A presente pesquisa foi submetida ao Comitê de Ética e Pesquisa da Universidade do Estado do Rio Grande do Norte, em atendimento à Resolução 196/96 do Conselho Nacional de Saúde e aprovada com número de parecer 563.599.

Foram realizadas coletas de queijos tipo coalho em sete queijarias artesanais informais localizadas no município de São Rafael (RN), que produziam queijo de coalho a partir do leite cru, não pasteurizado. Após a coleta, as amostras foram acondicionadas em caixas isotérmicas em gelo e transportadas imediatamente ao Laboratório de Biotecnologia de Alimentos do Centro de Ciências Agrárias da Universidade Federal Rural do Semi-Árido, onde as amostras foram submetidas as análises microbiológicas.
As amostras de cada queijaria foram analisadas em triplicata após o armazenamento a $7^{\circ} \mathrm{C}$ durante cinco dias depois da fabricação, permitindo um período de refrigeração e estocagem na prateleira para avaliação de qualidade. As análises microbiológicas foram realizadas de acordo com as recomendações da Instrução Normativa ${ }^{\circ}$ 62, de 26 de agosto de 2003, do Ministério da Agricultura, Pecuária e Abastecimento (BRASIL, 2003)

$\mathrm{Na}$ contagem de Staphylococcus aureus coagulase positivo foi utilizada a técnica de plaqueamento em superfície em Agar Baird-Parker adicionado de telurito de potássio a $1 \%$ e emulsão de gema de ovo, com posterior contagem do número de colônias típicas e isolamento em Agar Nutriente inclinado, realizando-se em seguida teste de coagulase. Os resultados foram expressos em Log de Unidades Formadoras de Colônias por grama da amostra (UFC/g) (BRASIL, 2003).

$\mathrm{Na}$ pesquisa de Salmonella sp. realizou-se o préenriquecimento em água peptonada, enriquecimento seletivo em Caldo Tetrationato e Caldo Selenito Cistina. Em seguida, procedeu-se o plaqueamento em Ágar Bismuto Sulfito e Agar Entérico Hektoen. Colônias típicas foram isoladas em Agar nutriente, e, posteriormente, realizados testes bioquímicos confirmatórios. Os resultados foram expressos como presença ou ausência de Salmonella em $25 \mathrm{~g}$ de queijo (BRASIL, 2003).

\section{RESULTADOS E DISCUSSÃO}

A Tabela 1 apresenta os resultados em log UFC/g para Staphylococcus aureus e ausência ou presença para Salmonella sp. em 25 gramas da amostra, obtidos a partir da contagem microbiológica das amostras de queijo de coalho.

Tabela 1. Resultados das análises microbiológicas para amostras de queijo de coalho (Q) produzidas artesanalmente no município de São Rafael, Rio Grande do Norte.

\begin{tabular}{lcccccccc}
\multicolumn{1}{c}{ Análises } & Q1 & Q2 & Q3 & Q4 & Q5 & Q6 & Q7 & Padrão* \\
\hline $\begin{array}{l}\text { S. aureus } \\
\text { (Log de }\end{array}$ & 6,66 & 4,84 & 5,92 & 4,52 & 6,08 & 5,30 & 5,85 & 2,70 \\
$\begin{array}{l}\text { UFC/g) } \\
\begin{array}{l}\text { Salmonella } \\
\text { sp. }\end{array}\end{array}$ & A & A & A & A & A & A & A & A \\
\hline
\end{tabular}

(*) Padrão RDC $\mathrm{n}^{\circ} 12$ de 2001: Dispõe sobre padrões microbiológicos sanitários para alimentos. $(\mathrm{A})=$ ausente

Todas as amostras apresentaram elevadas contagens de Staphylococcus aureus (Tabela 1).

O limite máximo permitido de Staphylococcus aureus é de $5 \times 10^{2} \mathrm{UFC} / \mathrm{g}$ (BRASIL, 2001), ou seja, 2,70 log de UFC. Observa-se na tabela 1 , que as sete amostras avaliadas apresentaram contagens acima deste valor.

Segundo Silva et al. (2010), os alimentos podem constar uma extensa variação e quantidade de micro-organismos que podem alterar a vida de prateleira ou veicular doenças a quem os consomem.

Os resultados encontrados no presente estudo podem indicar a falta de qualidade higiênico sanitária das amostras de queijo de coalho produzidas no município de São Rafael (RN), já que a presença de $S$. aureus pode estar relacionada à manipulação inadequada dos alimentos (SILVA et al., 2017).

Vários fatores relacionados ao manipulador podem favorecer a contaminação dos alimentos por microorganismos como os do grupo Staphylococcus, tais como o uso de adornos contaminados e a manipulação dos alimentos 
sem máscara e luvas. Isso por que essas bactérias são habitantes naturais do trato respiratório humano (FORSYTHE, 2013).

Além desses fatores, os hábitos de higiene pessoal do manipulador são as principais causas de propagação de doenças transmitidas por alimentos (SOUZA et al., 2017), como é o caso da contaminação do alimento por $S$. aureus através da mão do manipulador (SILVA et al., 2017).

A contaminação por essa bactéria tem alta prevalência em produtos láticos, principalmente queijos artesanais (BORGES et al., 2008), o que, possivelmente, explica os resultados detectados no presente estudo.

Resultados aproximados foram encontrados por Silva et al. (2010) em estudo sobre a influência dos procedimentos de fabricação nas características físico-químicas, sensoriais e microbiológicas de queijo de coalho. Esses autores detectaram valores de $2,0 \times 10^{4}$ a $4,0 \times 10^{7} \mathrm{UFC} / \mathrm{g}$ para Staphylococcus aureus. Santana et al. (2008), em trabalho sobre qualidade microbiológica de queijo de coalho comercializado em Aracaju (SE) detectaram Staphylococcus aureus em 46,7\% das 60 amostras analisadas em contagens de até 3,54 x $10^{5} \mathrm{UFC} / \mathrm{g}$. Feitosa et al. (2003) também verificaram altas contagens de Staphylococcus aureus na maioria dos queijos de coalho avaliados em sua pesquisa com queijos do Rio Grande do Norte.

Sousa et al. (2014), ao avaliarem a qualidade microbiológica de queijos de coalho provenientes de seis estados do Nordeste, detectaram altas contagens de Staphylococcus aureus em 95,16\% das amostras analisadas, verificando ainda presença deste patógeno em amostras de todos os estados avaliados. Esses autores em sua pesquisa chamam atenção em seu trabalho para o armazenamento em temperaturas inadequadas do queijo de coalho, que pode favorecer o crescimento microbiano e, consequentemente, diminuir a qualidade do produto final.

Evangelista-Barreto et al. (2016) detectaram elevadas contagens de S. aureus em $80 \%$ dos queijos de coalho analisados em seu trabalho com amostras provenientes da cidade de Cruz das Almas, Bahia.

Não foi encontrada Salmonella sp. nos queijos de coalho analisados na presente pesquisa, ou seja, as amostras pesquisadas neste trabalho apresentaram-se de acordo com a legislação vigente no que diz respeito à Salmonella, ou seja, ausência em 25 gramas.

O presente trabalho teve resultados próximos ao trabalho de Sousa et al. (2014) que encontraram resultados positivos para Salmonella em apenas uma amostra de um total de 104 avaliadas e Evangelista-Barreto et al. (2016) que constataram ausência de Salmonella em queijos de coalho comercializados em Cruz das Almas, Bahia. Enquanto Santana et al. (2008), em seu trabalho sobre qualidade microbiológica de queijo de coalho comercializado em Aracaju-SE, detectaram presença de Salmonella sp. (em 25g) em 26,7\% das 60 amostras de queijo analisadas.

Como a pasteurização destrói a grande maioria dos micro-organismos presentes no leite (GAVA, 2010), há uma probabilidade maior de ocorrência de Salmonella em queijos fabricados com leite cru (SOUSA et al., 2014). Na fabricação do queijo de coalho tradicional utiliza-se leite cru, mas durante o dessoramento do coágulo é feito o aquecimento normalmente a temperaturas acima de $55^{\circ} \mathrm{C}$ ou o cozimento do queijo em soro quente, o que, possivelmente, poderia justificar a ausência de Salmonella, visto que são micro- organismos com pouca resistência ao calor. Ou seja, não é por que não foi encontrada a Salmonella que o queijo de coalho foi produzido em boas condições higiênico sanitárias nas queijarias.

\section{CONCLUSÕES}

Os queijos de coalho produzidos artesanalmente no município de São Rafael, Rio Grande do Norte, evidenciaram condições de higiene inadequadas, apresentando contaminação elevada por Staphylococcus aureus, e consequentemente, não se enquadraram dentro dos parâmetros definidos pela legislação. Assim, todos os queijos de coalho foram classificados como impróprios para o consumo humano.

\section{REFERÊNCIAS}

BORGES, M. F.; ARCURI, E.F.; PEREIRA, J.L.; FEITOSA, T.; KUAYE, A.Y. Staphylococcus enterotoxigênicos em leite e produtos lácteos, suas enterotoxinas e genes associados: revisão. Boletim do Centro de Pesquisa e Processamento de Alimentos, v. 26, n. 1, p. 71-86, 2008

BORGES, M. F.; FEITOSA, T.; NASSU, R. T.; MUNIZ, C. R.; AZEVEDO, E. H. F.; FIGUEIREDO, A. T. Microrganismos patogênicos e indicadores em queijo coalho produzido no Estado do Ceará, Brasil. Boletim do Centro de Pesquisa e Processamento de Alimentos, v.21, n.1, p.31- 40, 2003.

BRASIL. Ministério da Agricultura, Pecuária e Abastecimento. Instrução Normativa $\mathrm{n}^{\circ}$ 62, de 26 de agosto de 2003. Diário Oficial da União. Brasília, 18 de setembro de 2003.

BRASIL. Resolução RDC n ${ }^{\circ} 12$, de 02 de janeiro de 2001 da Agencia Nacional de Vigilância Sanitária do Ministério da Saúde. Dispõe Sobre Padrões Microbiológicos Sanitários para Alimentos. Diário Oficial [da] Republica Federativa do Brasil, Brasília, DF. 10 jan. 2001. Seção 1, no 7-E, p.1415-53.

EVANGELISTA-BARRETO, N. S.; SANTOS, G. C. F.; SOUZA, J. S.; BERNARDES, F. S.; SILVA, I. P. Queijos artesanais como veiculo de contaminação de Escherichia coli e estafilococos coagulase positiva resistentes a antimicrobianos. Revista Brasileira de Higiene e Sanidade Animal, v.10, n. 1, p. 55-67, 2016.

FEITOSA, T.; BORGES, M. F.; NASSU, R.T.; AZEVEDO, E. H. F.; MUNIZ, C. R. Pesquisa de Salmonella sp., Listeria sp. e microrganismos indicadores higiênico-sanitários em queijos produzidos no estado do Rio Grande do Norte. Ciência e Tecnologia de Alimentos, v. 23, n.3, 2003.

FORSYTHE, S. T. Microbiologia da segurança do alimentos. 2 ed. Porto Alegre: Artmed, 2013.

GAVA, A. J. Tecnologia de alimentos: princípio e aplicações. Barueri: Nobel, 2010, 512p. 
GERMANO, P. M. L.; GERMANO, M. I. S. Higiene e vigilância sanitária de alimentos. $3^{\mathrm{a}}$ edição. Barueri: Manole, 2008, 986p.

JAY, J. M. Microbiologia de Alimentos. $6^{\text {a }}$ Ed. Porto Alegre: ARTEMD, 2005.

MACHADO, P. F.; LIMA, U. A. LEITE. In: LIMA, U. A. Matérias-primas dos alimentos. São Paulo: Editora Blucher, 2010, 402p.

MIRANDA, G. R.; SOUZA, A. M.; MARTINS, A. D. O.; COCARO, E. S.; MARTINS, J. M. Queijos artesanais: qualidade físico-química e microbiológica e avaliação das condições higiênico-sanitárias dos manipuladores e ambiente de produção. Extensão Rural, v.23, n.1, p.78-92, 2016.

OLIVEIRA K. D.; EVENCIO NETO J.; PAIVA J. E Qualidade microbiológica do queijo de coalho comercializado no Município do Cabo de Santos Agostinho, Pernambuco, Brasil. Arquivos do Instituto Biológico, v. 77, n. 3, p. 435440, 2010.

SANTANA, R. F.; SANTOS, D. M.; MARTINEZ, A. C. C.; LIMA, A. S. Qualidade microbiológica de queijo-coalho comercializado em Aracaju, SE. Arquivo Brasileiro de Medicina Veterinária e Zootecnia, v.60, n.6, 2008.

SCHIMITT, C. I.; CERESER, N. D.; BOHRZ, D. A. S.; NOSKOSKI, L. Contaminação do queijo colonial de produção artesanal comercializado em mercados varejistas do Rio Grande do Sul. Veterinária Notícias, v 17, n 2, 2011.

SILVA, C. A.; FERNANDES, A. R. Projetos de empreendimentos agroindustriais: produtos de origem animal. Vol 1. Viçosa: Editora UFV, 2011, 308p.

SILVA, M. C. D.; RAMOS, A. C. S.; MORENO, I.; MORAES, J. O. Influência dos procedimentos de fabricação nas características físico-químicas, sensoriais e microbiológicas de queijo de coalho. Revista do Instituto Adolfo Lutz, v.69, n.2, p.214-21, 2010.

SILVA, R.N.A.; SANTOS, A.P.L.; SOARES, L.S. Avaliação microbiológica das mãos de manipuladores em restaurantes comerciais e institucionais da cidade de Salvador, BA. Higiene Alimentar, v.31, n 270/271, 2017.

SOUSA, A. Z. B.; ABRANTES, M. A.; SAKAMOTO, S. M.; SILVA, J. B. A.; LIMA, P. O.; LIMA, R. N.; ROCHA, M. O. C.; PASSOS, Y. D. B. Aspectos físico-químicos e microbiológicos do queijo tipo coalho comercializado em estados do nordeste do Brasil. Arquivos do Instituto Biológico, v.81, n.1, p.30-35, 2014.

SOUZA, P. S.; GONÇALVES, S. A.; DIAS, R. F. Aplicação de boas práticas por manipuladores de alimentos em pontos de venda de gelados comestíveis. Higiene Alimentar, n. 31, ${ }^{\circ}$ 268/269, 2017. 